\section{Évaluation de la protection vaccinale contre la peste bovine au Cameroun}

\section{La province du Nord}

\author{
A. Ngangnou ${ }^{1}$ \\ N. Zoyem ${ }^{1}$
}

NGANGNOU (A.), ZOYEM (N.). Évaluation de la protection vaccinale contre la peste bovine au Cameroun. II. La province du Nord. Revue Élev. Méd. vét. Pays trop., 1995, 48 (3) : 236-238.

Le Cameroun participe à la Campagne panafricaine de lutte contre la peste bovine (PARC) depuis 1989 (dans son volet séro-surveillance des anticorps bovipestiques). Durant la campagne de 1992, un protocole d'échantillonnage adapté aux conditions d'élevage dans la province du Nord a été élaboré en conformité avec les recommandations de la PARC. Les quatre départements de la province ont été couverts. Quatorze sites ont été choisis par la technique de l'échantillonnage aléatoire et visités. Huit mille six cent quatre-vingt échantillons de sérums issus de 217 troupeaux ont été prélevés et testés par la technique ELISA compétition développée et adaptée à la peste bovine par le projet conjoint FAO/AIEA. Les résultats obtenus montrent une prévalence générale de $66 \mathrm{p} .100$, ce qui est en dessous du seuil requis pour une bonne protection vaccinale du cheptel. Les différences de prévalence entre les tranches d'âge et entre les troupeaux transhumants et nomades sont statistiquement significatives. Ces résultats, bien qu'identiques à ceux observés l'année précédente dans la province de l'Adamaoua (62 p. 100 en 1991), ne reflètent pas la situation sur toute l'étendue du territoire national. Une concertation entre les différents responsables des provinces à forte densité de bovins est recommandée, afin d'adopter les mesures et les stratégies communes de vaccination avec pour objectif principal l'amélioration du niveau de la couverture vaccinale.

Mots-clés : Bovin - Peste bovine - Enquête - Vaccin - Test ELISA Cameroun.

\section{Introduction}

Dans le cadre de la Campagne panafricaine de lutte contre la peste bovine (PARC), le Laboratoire national vétérinaire de Garoua-Boklé a entrepris pour la deuxième année consécutive, une enquête sérologique, en vue d'estimer le taux de protection vaccinale des bovins au Cameroun.

La province du Nord dans laquelle l'enquête a eu lieu en 1992, couvre une superficie de $65000 \mathrm{~km}^{2}$, soit 17,3 p. 100 du territoire national avec 651200 têtes de bovins, soit 15 p. 100 de l'effectif total (5).

Le cheptel bovin de cette région est composé de taurins (dans le département du Faro), de zébus Bororos (dans les départements de la Bénoué et du Mayo-Rey), de zébus Bokolos (dans le département du Mayo-Rey) et de Goudalis ou zébus Peuhls de petite taille (dans le Mayo-

1. Laboratoire national vétérinaire (LANAVET), BP 503, Garoua, Cameroun.

Reçu le 18.6.1993, accepté le 7.11.1995.
Rey, en particulier dans les zones voisines de I'Adamaoua) (5).

\section{Matériel et Méthode}

\section{Échantillonnage}

Dans les 8 sous-secteurs de la province, 14 sites correspondant aux centres zootechniques et vétérinaires ont été choisis par la technique d'échantillonnage aléatoire, à raison de 6 sites par sous-secteur. Dans les sous-secteurs ayant moins de six sites, un coefficient de pondération a été adopté : $\mathrm{N}=0,75 * \mathrm{n}$ ( $\mathrm{N}$ est le nombre de sites à choisir, $n$ le nombre de sites dénombrés) (7).

Dans chaque site, les troupeaux dans lesquels les prélèvements doivent être effectués ont été choisis par randomisation et libellés en deux rubriques: les principaux et les substituts (destinés au remplacement des troupeaux partis en transhumance lors du passage des équipes de prélèvement).

\section{Prélèvements}

Quarante prélèvements ont été effectués dans chaque troupeau, répartis de la manière suivante:

- 10 prélèvements sur des sujets âgés de moins d'un an ;

- 10 sur des sujets de un à deux ans ;

- 10 sur des sujets âgés de deux à trois ans ;

- 10 sur des sujets âgés de plus de trois ans.

L'âge est déterminé par l'interrogatoire du propriétaire et l'examen de la dentition. Les tubes sont identifiés par un code spécial défini par la PARC (2) : ce code indique le secteur, le sous-secteur, le site, le propriétaire du troupeau, l'âge et le sexe. Le type d'élevage (sédentaire ou transhumant) a été mentionné sur les fiches épidémiologiques.

Les sérums ont été récoltés 24 à 36 heures après prélèvement, transportés au laboratoire sous glace dans des coffres isothermes et gardés à $-20^{\circ} \mathrm{C}$ en attendant d'être testés.

La méthode utilisée est l'ELISA competition (Enzyme Linked Immuno-Sorbant Assay) développée et adaptée à la peste bovine par le projet conjoint FAO/AIEA. Les sérums sont testés à la dilution de $1 / 10(1,3,4)$.

\section{Résultats}

La figure 1 montre l'emplacement géographique des 14 sites visités. Les tableaux I, II et III, présentent les résultats obtenus en fonction respectivement du secteur, des tranches d'âge et du mode d'élevage. 


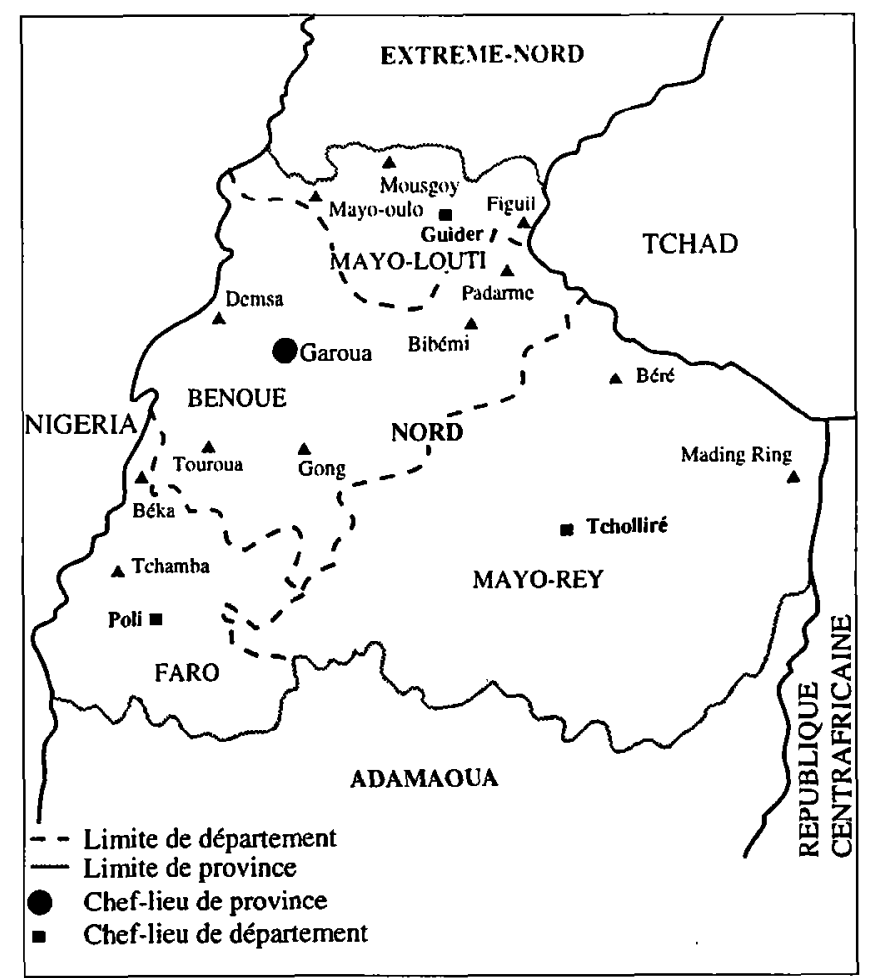

Figure 1 : Province du Nord : sites choisis.

\section{La province}

La prévalence globale est de 66 p. $100 \pm 1$ Les résultats enregistrés la même année dans les pays voisins sont les suivants: Tchad, 45,3 p. 100 ; République centrafricaine, 57 p. 100 ; Nigeria, 52 p. 100 (6).

\section{Les secteurs}

Le tableau I montre qu'il existe une différence significative de taux de séro-positivité entre les départements de la Bénoué et du Faro (chi-2 = 30,59; d.d.I = 1; $p<0,05$ ). Par contre, elle n'est pas significative entre les départements du Mayo-Rey et du Mayo-Louti (chi-2 = 3,30; d.d.I $=1 ; p>0,05$ ).

\section{Les différentes tranches d'âge}

Le tableau II montre que le taux de séro-positivité est inférieur à 50 p. 100 chez les sujets âgés de moins d'un an : il s'accroît considérablement entre un et trois ans.

Il existe une différence significative des taux de séropositivité entre les trois tranches d'âge, 1-2 ans, 2-3 ans, supérieure à 3 ans (chi-2 $=85,23$; d.d.l $=2 ; p<0,05$ ); il en est de même entre les tranches d'âge de $2-3$ ans et supérieurs à 3 ans (chi-2 = 20,76; d.d.I = $1 ; p<0,05$ ).
Tableau I

Répartition des résultats par secteur

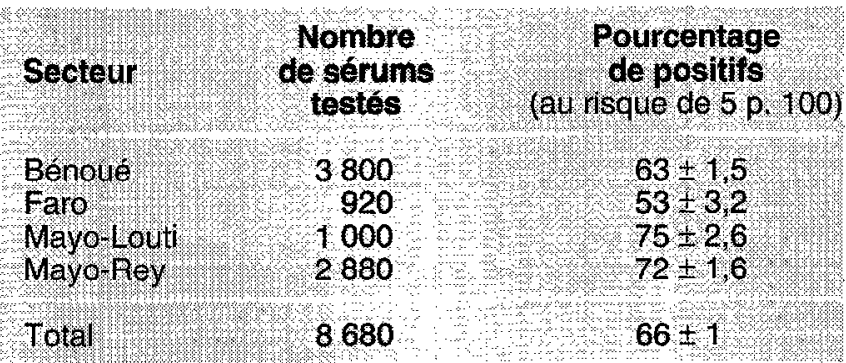

Tableau II

Répartition des résultats par tranche d'âge

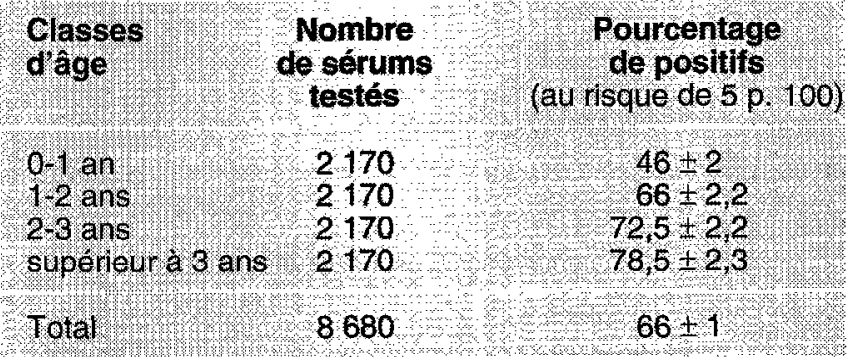

Tableau III

Répartition des résultats par mode d'élevage

\begin{tabular}{|c|c|c|c|c|}
\hline \multirow{2}{*}{ cte } & \multicolumn{2}{|c|}{$\begin{array}{l}\text { Nombre } \\
\text { de sérums } \\
\text { testés }\end{array}$} & \multicolumn{2}{|c|}{$\begin{array}{l}\text { Pourcentage } \\
\text { de positifs } \\
\text { (au rsque de } 5 \text { p. } 100)\end{array}$} \\
\hline & $\begin{array}{l}\text { Elevage } \\
\text { nshumant }\end{array}$ & $\begin{array}{l}\text { Elevage } \\
\text { sédentaire }\end{array}$ & $\begin{array}{l}\text { Ele } \\
\text { rans! }\end{array}$ & sed \\
\hline $\begin{array}{l}\text { ne } \\
\text { Louti } \\
\text {-Rey }\end{array}$ & $\begin{array}{r}2820 \\
920 \\
1000 \\
600\end{array}$ & $\begin{array}{r}1560 \\
20 \\
2280\end{array}$ & $\begin{array}{l}44+1,5 \\
53+3,2 \\
75+1,6 \\
70 \pm 1\end{array}$ & $\begin{array}{l}82+1 \\
0 \\
0 \\
74+1\end{array}$ \\
\hline & & & $00 \pm$ & \\
\hline
\end{tabular}

\section{Le mode d'élevage}

Le tableau III montre que les troupeaux sédentaires sont mieux protégés que les nomades (chi- $2=388,14 ; \mathrm{d} . \mathrm{d} . \mathrm{I}=$ $1 ; p<0,05)$.

\section{Discussion}

La prévalence globale est presque identique à celle enregistrée dans la province de l'Adamoua en 1991 (62 p. 100) (8). 
Ces résultats sont inquiétants. En effet, en raison des mouvements de transhumance entre les secteurs de la Bénoué et du Faro d'une part (surtout de Gong vers Béka et Tchamba) et d'autre part, entre le Mayo-Louti et le Mayo-Rey, le faible taux d'animaux immuns permettrait à un foyer potentiel de s'étendre rapidement et sur une grande étendue de la région.

Plusieurs raisons peuvent être avancées pour justifier ces résultats :

1. les jeunes de moins d'un an échappent souvent à la vaccination en raison de la transhumance, ce qui a conduit à proposer un élargissement des campagnes de vaccination aux jeunes de moins d'un an (avant et au retour des transhumances, si le coût financier le justifie) (9) ;

2. le manque de moyens financiers en vue du renforcement de la chaîne de froid et de l'achat du matériel de vaccination, à savoir l'acquisition des congélateurs, des glacières, des seringues, des aiguilles, réduit considérablement l'efficacité des campagnes de vaccination ;

3. en l'absence du diluant approprié (le sulfate de magnésium molaire), les vaccins sont le plus souvent reconstitués avec de l'eau de pluie refroidie et réchauffée ;

4. les parcours de vaccination sont souvent vétustes, ce qui ne permet pas une contention adéquate des troupeaux durant les séances de vaccination.

\section{Conclusion}

Durant la campagne de séro-surveillance de la peste bovine de 1992 dans la province du Nord, 8680 sérums ont été prélevés et testés. Les résultats montrent que le seuil de protection immunitaire du cheptel n'est pas encore atteint ( 68 p. 100 de sérums testés présentent des anticorps bovipestiques). Ces résultats sont similaires à ceux obtenus dans la province de l'Adamoua (62 p. 100, campagne de 1991).

L'obtention de résultats satisfaisants passe par l'instauration d'une politique de concertation entre les responsables des campagnes de vaccination des provinces à forte densité de cheptel bovin, dans le but de redynamiser celles-ci.
Un accent doit être mis sur : la recherche des voies et moyens pour construire les parcs de vaccination (là où il n'y en a pas) et rénover ceux existants ; l'acquisition du matériel de vaccination; l'observation rigoureuse des règles de conservation et d'administration du vaccin (reconstitution, voie d'inoculation); dans l'ignorance de l'immunité des mères et donc de celle récupérée par le veau, il est préférable de vacciner tous les veaux, sans toutefois les marquer à l'oreille : ainsi peut-on espérer rompre le cycle du virus (9).

\section{Remerciements}

Ce travail a été financé par le Fonds européen de développement (FED) que nous remercions grandement. Notre gratitude va aussi à la division AIEA/FAO pour la fourniture des trousses de recherches d'anticorps bovipestiques. Nous remercions également le Dr Lise Guerre pour son aide lors de la rédaction de manuscrit.

\section{Bibliographie}

Sera publiée dans le troisième et dernier article.

NGANGNOU (A.), ZOYEM (N.). Evaluation of the vaccinal protection against rinderpest in Cameroon. II. The North Province. Revue Elev. Méd. vét. Pays trop., 1995, 48 (3) : 236-238.

Cameroon joined the sero-survey component of the PARC (Pan African Rinderpest Campaign) program in 1989. During the 1992 Campaign, a detailed sampling frame, adapted to the breeding conditions of the North Province was drawn up according to PARC recommendations. The four administrative divisions of the province were covered by sampling cattle in 14 sites chosen by randomisation. Eight thousand six hundred and eighty sera samples from 217 cattle herds were tested using FAO/IAEA rinderpest competitive ELISA technique. The results indicated an overall prevalence of rinderpest virus antibodies (RPVA) of $66 \%$. This is below the target objective. The differences of prevalence between age groups and breeding systems (sedentary or transhumant) are statistically significant. The same results have been reported in the Adamaoua Province ( $62 \%$ in 1991 campaign). These results do not reflect the situation in all the country. It is suggested to hold general meeting between differen livestock managers from the provinces with high cattle populations to adopt commun vaccination measures with the target objective of increasing the level of immunity.

Key words : Cattle - Rinderpest - Survey - Vaccine - ELISA - Cameroon. 